\title{
Perturbative computation of glueball superpotentials
}

\section{Citation}

Dijkgraaf, R., M.T. Grisaru, C.S. Lam, C. Vafa, and D. Zanon. 2003. “Perturbative Computation of Glueball Superpotentials." Physics Letters B 573 (October): 138-46. https://doi.org/10.1016/ j.physletb.2003.08.060.

\section{Permanent link}

http://nrs.harvard.edu/urn-3:HUL.InstRepos:41385005

\section{Terms of Use}

This article was downloaded from Harvard University's DASH repository, and is made available under the terms and conditions applicable to Other Posted Material, as set forth at http:// nrs.harvard.edu/urn-3:HUL.InstRepos:dash.current.terms-of-use\#LAA

\section{Share Your Story}

The Harvard community has made this article openly available.

Please share how this access benefits you. Submit a story.

Accessibility 
hep-th/0211017

HUTP-02/A056

ITFA-2002-47

McGill/02-137

IFUM-734-FT

\title{
Perturbative Computation of Glueball Superpotentials
}

\author{
R. Dijkgraaf, ${ }^{1}$ M.T. Grisaru, ${ }^{2}$ C.S. Lam,${ }^{2}$ C. Vafa,${ }^{3}$ and D. Zanon ${ }^{4}$ \\ ${ }^{1}$ Institute for Theoretical Physics 83 Korteweg-de Vries Institute for Mathematics, \\ University of Amsterdam, 1018 XE Amsterdam, The Netherlands \\ 2 Physics Department, McGill University, \\ Montreal, QC Canada H3A 2T8 \\ 3 Jefferson Physical Laboratory, Harvard University, \\ Cambridge, MA 02138, USA \\ ${ }^{4}$ Dipartimento di Fisica Dell'Universitá di Milano, \\ INFN, Sezione di Milano, Via Celoria 16, I-20133 Milano
}

Using $\mathcal{N}=1$ superspace techniques in four dimensions we show how to perturbatively compute the superpotential generated for the glueball superfield upon integrating out massive charged fields. The technique applies to arbitrary gauge groups and representations. Moreover we show that for $U(N)$ gauge theories admitting a large $N$ expansion the computation dramatically simplifies and we prove the validity of the recently proposed recipe for computation of this quantity in terms of planar diagrams of matrix integrals.

November, 2002 


\section{Introduction}

The aim of this note is to use superspace techniques to compute the glueball superpotential in perturbation theory in gauge theories with $\mathcal{N}=1$ supersymmetry. In particular we will show that this computation confirms the general prescription proposed in [1] that these perturbative calculations can be done, for theories admitting a large $N$ expansion, in a zero-dimensional reduction to a matrix model. This conjecture has up to now only been proven in special cases where the corresponding theory could be engineered in string theory.

In this paper we basically make precise the field theory arguments sketched in [1] by showing that the computations of the superspace Feynman graphs for a chiral superfield in an external gauge field reduce to a zero-dimensional path-integral. However, unlike in [1], we will not limit ourselves to the case where the theory has a large $N$ expansion. Instead we set up the computation for an arbitrary $\mathcal{N}=1$ supersymmetric gauge theory coupled to arbitrary matter. Moreover we show that for the case of $U(N)$ gauge theories admitting a large $N$ description this leads to the recipe advanced in [1]. The simplifications for other gauge groups and more general representations will appear in a forthcoming paper [2]. This leads to exact computation of F-terms for $\mathcal{N}=1$ supersymmetric gauge theories in four dimensions for arbitrary gauge group and matter content, upon extremization of the glueball superpotential.

The organization of this note is as follows: In section 2 we briefly review the relevant superspace techniques [3]. In section 3 we set up the general computation for a general gauge group coupled to matter. In section 4 we note the simplification in the context of $U(N)$ gauge theories coupled to matter admitting a large $N$ expansion and confirm the proposal of [1]. We illustrate the proof by working out a concrete example.

\section{Chiral Superspace Techniques}

We consider the action, in the presence of an external gauge field, for a self-interacting massive chiral superfield $\Phi$ in some representation of the gauge group

$$
S(\Phi, \bar{\Phi})=\int d^{4} x d^{4} \theta \bar{\Phi} e^{V} \Phi+\int d^{4} x d^{2} \theta W(\Phi)+\text { h.c. }
$$

where we used a gauge invariant pairing between $\Phi$ and $\bar{\Phi}$, and where $W(\Phi)$ is some gauge invariant superpotential. We use the conventions from [3] and in particular, for the gauge field strength we have

$$
\mathcal{W}_{\alpha}=i \bar{D}^{2} e^{-V} D_{\alpha} e^{V}
$$


and a corresponding expression for $\overline{\mathcal{W}}_{\dot{\alpha}}$. Here $\bar{D}^{2}=\frac{1}{2} \bar{D}^{\dot{\alpha}} \bar{D}_{\dot{\alpha}}$ and similarly $D^{2}=\frac{1}{2} D^{\alpha} D_{\alpha}$. The covariant derivatives can be chosen as $D_{\alpha}=\partial / \partial \theta^{\alpha}, \bar{D}_{\dot{\alpha}}=\partial / \partial \bar{\theta}^{\dot{\alpha}}+i \bar{\theta}^{\dot{\alpha}} \partial_{\alpha \dot{\alpha}}$. Other superspace facts are the relations

$$
\bar{D}^{2} D^{2} \Phi=\square \Phi, \quad D^{2} \bar{D}^{2} \bar{\Phi}=\square \bar{\Phi}
$$

valid for (anti)chiral superfields. Here we consider, for simplicity of presentation, a single gauge group, but the analysis can be easily extended to products of gauge groups as well.

Perturbative calculations for this system are best carried out in the background field method and details can be found in [3]. Furthermore, one can develop covariant supergraph techniques [4] which extend and simplify ordinary supergraph calculations. Here we will not need the full power of this method; we will rederive what is necessary for the computation of the glueball superpotential.

In the usual supergraph analysis for the present system one has to deal with propagators $\langle\Phi \Phi\rangle,\langle\overline{\Phi \Phi}\rangle$ and $\langle\Phi \bar{\Phi}\rangle$. This would be true if one is trying to compute an arbitrary amplitude. However, here we are interested in certain F-terms and as we now explain (and can also be verified using covariant supergraph techniques [4]) only holomorphic propagators $\langle\Phi \Phi\rangle$ contribute to these terms.

The argument for this is rather simple and well-known: terms appearing in the superpotential cannot depend on the coefficients of the anti-chiral superpotential $\bar{W}(\bar{\Phi})$. This is so because we can promote each of those coefficients to an anti-chiral field, whose vev then gives the coupling constants. Holomorphy tells us that these fields cannot appear in an integral over chiral superspace. Since we are interested in computing the superpotential for a chiral glueball superfield, we thus know that the antichiral coefficients do not contribute to this amplitude.

We use this fact to choose a simple form for $\bar{W}(\bar{\Phi})$ which is not necessarily the complex conjugate of $W(\Phi)$. (Holomorphy allows us to treat them independently.) Moreover, we assume that the fields are such that we can give them a bare mass. For simplicity, say, $\Phi$ is taken to transform in a real representation so we can have a gauge invariant (necessarily chiral) mass term $\frac{m}{2} \Phi^{2}$. For $\bar{W}$ we now take the simple form

$$
\bar{W}(\bar{\Phi})=\frac{1}{2} \bar{m}^{2}
$$

(We could have also restricted to $\bar{W}=0$ and we will comment on that possibility below). With this choice, the $\bar{\Phi}$ action is quadratic and the antichiral superfield $\bar{\Phi}$ can be integrated out, leaving us to deal only with the chiral superfield $\Phi$. 
It is economical, in considering the kinetic term $\bar{\Phi} e^{V} \Phi$ to define the covariantly antichiral superfield

$$
\tilde{\Phi}=\bar{\Phi} e^{V}=e^{-V} \bar{\Phi}
$$

(the last identity holds for real representations). In this so-called gauge chiral representation [3] this field is trivially annihilated by the covariant derivative $\nabla_{\alpha}=e^{-V} D_{\alpha} e^{V}$. The corresponding covariantly chiral superfield is identical to the ordinary chiral superfield and it is annihilated by the ordinary derivative $\bar{\nabla}_{\dot{\alpha}} \equiv \bar{D}_{\dot{\alpha}}$. We have identities such as $\bar{\nabla}^{2} \nabla^{2} \Phi=\square_{+} \Phi$ where, by straightforward algebra,

$$
\square_{+} \Phi=\left[\square_{\mathrm{cov}}-i \mathcal{W}^{\alpha} \nabla_{\alpha}-\frac{i}{2}\left(\nabla^{\alpha} \mathcal{W}_{\alpha}\right)\right] \Phi
$$

There is a corresponding relation $\nabla^{2} \bar{\nabla}^{2} \tilde{\Phi}=\square_{-} \tilde{\Phi}$ with $\nabla^{2} \square_{+}=\square_{-} \bar{\nabla}^{2}$. In the expression above $\square_{\text {cov }}=\frac{1}{2} \nabla^{\alpha \dot{\alpha}} \nabla_{\alpha \dot{\alpha}}$ and $\nabla_{\alpha \dot{\alpha}}=-i\left\{\nabla_{\alpha}, \bar{\nabla}_{\dot{\alpha}}\right\}$.

After these preliminaries we consider integrating out the antichiral field from the action

$$
S(\Phi, \bar{\Phi})=\int d^{4} x d^{4} \theta \tilde{\Phi} \Phi+\int d^{4} x d^{2} \theta W(\Phi)+\int d^{4} x d^{2} \bar{\theta} \frac{\bar{m}}{2} \tilde{\Phi}^{2} .
$$

Note that since the antichiral mass term must be a gauge singlet we were allowed to replace the quadratic term $\bar{\Phi}^{2}$ by $\tilde{\Phi}^{2}$ (e.g., consider the case of the adjoint representation where $\bar{\Phi}$ is an adjoint matrix, $\tilde{\Phi}=e^{-V} \bar{\Phi} e^{V}$ and the $V$-dependence drops out of $\operatorname{Tr} \tilde{\Phi}^{2}$.) In standard fashion we wish to complete the square, but first we rewrite

$$
\int d^{4} x d^{2} \bar{\theta} \tilde{\Phi}^{2}=\int d^{4} x d^{4} \theta \tilde{\Phi} \frac{1}{\square_{+}} \bar{\nabla}^{2} \tilde{\Phi}
$$

which can be checked by the replacement $\int d^{2} \theta \rightarrow D^{2}=\nabla^{2}$ when acting on a gauge singlet.

We now complete the square as

$$
\int d^{4} x d^{4} \theta\left\{\frac{\bar{m}}{2}\left[\tilde{\Phi}+\frac{1}{\bar{m}} \nabla^{2} \Phi\right] \frac{1}{\square_{+}} \bar{\nabla}^{2}\left[\tilde{\Phi}+\frac{1}{\bar{m}} \nabla^{2} \Phi\right]-\frac{1}{2 \bar{m}} \nabla^{2} \Phi \frac{1}{\square_{+}} \bar{\nabla}^{2} \nabla^{2} \Phi\right\} .
$$

We integrate by parts the $\nabla^{2}$ and by the identities written above get rid of the $1 / \square_{+}$. The antichiral superfield can be integrated out now. In the last term we replace $\int d^{2} \bar{\theta}$ by $\bar{\nabla}^{2}$ ending up with the action

$$
S(\Phi)=\int d^{4} x d^{2} \theta \frac{-1}{2 \bar{m}} \Phi\left[\square_{\mathrm{cov}}-i \mathcal{W}^{\alpha} \nabla_{\alpha}-\frac{i}{2}\left(\nabla^{\alpha} \mathcal{W}_{\alpha}\right)\right] \Phi+W(\Phi) .
$$


For the purpose of determining the effective potential of a constant singlet gaugino condensate $S \sim \mathcal{W}^{\alpha} \mathcal{W}_{\alpha}$ several simplifications are now possible:

(a) We can assume that $\mathcal{W}^{\alpha}$ is covariantly constant,

$$
\nabla_{\alpha \dot{\alpha}} \mathcal{W}^{\beta}=\partial_{\alpha \dot{\alpha}} \mathcal{W}^{\beta}-i\left[\Gamma_{\alpha \dot{\alpha}}, \mathcal{W}^{\beta}\right]=0
$$

This implies, in particular, that $\mathcal{W}$ commutes with $\square_{\text {cov }}$ and allows us to move it freely from one line to another in a loop, at least as far as the spacetime part is concerned.

(b) We can drop the term $\nabla^{\alpha} \mathcal{W}_{\alpha}$ which will never enter in a relevant F-term.

(c) We can go to a new gauge antichiral basis by rewriting

$$
\Phi\left[\square_{\mathrm{cov}}-i \mathcal{W}^{\alpha} e^{-V} D_{\alpha} e^{V}\right] \Phi=\Phi^{\prime}\left[\square_{\mathrm{cov}}^{\prime}-i \mathcal{W}^{\prime \alpha} D_{\alpha}\right] \Phi^{\prime}
$$

where $\Phi^{\prime}=e^{V} \Phi$ and $\mathcal{W}^{\prime}=e^{V} \mathcal{W} e^{-V}$. The functional integral for $\Phi^{\prime}$ is the same as for $\Phi$ (it is a local field redefinition) while in the gaugino condensate $\operatorname{Tr} \mathcal{W}^{2}=\operatorname{Tr} \mathcal{W}^{\prime 2}$.

(d) The connection terms in $\square_{\text {cov }}$ can be treated perturbatively and in general would serve to covariantize derivative terms in the effective action, of which there are none in the present situation. (This is not entirely true: spinor derivatives of the space-time connection can lead to field strengths but a more detailed covariant supergraph analysis shows that this is not the case here.) Therefore the connections can be dropped from $\square_{\text {cov }}$ and we can replace it with the ordinary d'Alembertian $\square$.

Summarizing, we can conclude that for our purpose of computing F-terms in an external gauge field the relevant action can be written as

$$
\int d^{4} x d^{2} \theta\left(\frac{-1}{2 \bar{m}} \Phi\left(\square-i \mathcal{W}^{\alpha} D_{\alpha}\right) \Phi+W_{\text {tree }}(\Phi)\right)
$$

\section{The Perturbative Computation}

The action (2.13) derived above will be our starting point for the perturbative computation of the effective superpotential in the $\mathcal{W}$ background. We will write the tree-level superpotential as

$$
W_{\text {tree }}(\Phi)=\frac{m}{2} \Phi^{2}+\text { interactions. }
$$

It is convenient to include the $m \Phi^{2}$ part of the superpotential in the propagators, as usual. 


\subsection{Localization and $\bar{m}$-independence}

One important aspect of the above action is the fact that the superfield $\mathcal{W}^{\alpha}$ is correlated with the spinor derivative $D_{\alpha}$, and this confirms the approach used in [1]. In [1] it was also suggested that localization of the path integral to constant modes arises by asking which configurations can contribute to a term that can be written as an integral over chiral superspace. One used the argument familiar from topological field theory that derivatives can be written as commutators with the antichiral supercharge $\bar{Q}_{\dot{\alpha}}$ and therefore do not contribute in a $\int d^{2} \theta$ term. Let us see if this latter conclusion is valid in the present setup.

As we discussed before, the above path-integral should be $\bar{m}$ independent. We will verify this directly below, in perturbation theory. Since the answer does not depend on $\bar{m}$, one could even take the limit $\bar{m} \rightarrow 0$, i.e. set the antichiral superpotential $\bar{W}(\bar{\Phi})=0$. Because $1 / \bar{m}$ multiplies the kinetic term in the Lagrangian (2.13), in this case the pathintegral over the chiral field $\Phi$ will localize to the solutions of

$$
\left(\square-i \mathcal{W}^{\alpha} D_{\alpha}\right) \Phi=0
$$

In the absence of a $\mathcal{W}^{\alpha}$ background this would imply (in a Euclidean path-integral) a localization to harmonic and therefore constant $\Phi$. However, for us it is crucial to have a non-vanishing $\mathcal{W}^{\alpha}$ background turned on. So we see that it is not quite correct to say that the localization to constant modes takes place when this background is turned on. There will be non-trivial $x$ (or $p$ ) dependence, although the solution space to the constraint (3.2) is still finite dimensional. One can then localize the path-integral near these configurations and compute the partition function. However, instead, it turns out to be easier to directly do the Feynman graphs of this theory.

Let us first show by direct computation why the $\bar{m}$ dependence cancels out. Consider a Feynman graph made of chiral superfields with a total of $\ell$ loops. We will have one 4-momentum integral $\int d^{4} p$ for each loop. Moreover, we can also go to a momentum representation in the $\theta^{\alpha}$-directions and write

$$
D_{\alpha}=\partial / \partial \theta^{\alpha}=-i \pi_{\alpha}
$$

leading to $2 \ell$ grassmann momentum integration variables.

The bosonic part of the propagator is $\bar{m} /\left(p^{2}+m \bar{m}\right)$ and we can remove its $\bar{m}$ dependence by rescaling $p^{2} \rightarrow \bar{m} p^{2}$. Then the bosonic $d^{4} p$ momentum integrals will rescale with a factor of $\bar{m}^{2 \ell}$. On the other hand, to absorb the fermionic momentum integrations $\int d^{2} \pi$ 
we need $2 \ell$ interaction factors $\mathcal{W}^{\alpha} \pi_{\alpha}$ from the action and each comes with a factor of $1 / \bar{m}$ from the action or, equivalently, since we expand the propagator

$$
\frac{\bar{m}}{p^{2}+m \bar{m}+\mathcal{W}^{\alpha} \pi_{\alpha}}
$$

in an external background. Thus we obtain a factor of $1 / \bar{m}^{2 \ell}$ from the fermionic momentum integration and we see that the factors of $\bar{m}$ cancel between bosonic and fermionic momentum integrations. Below we will use this freedom to scale $\bar{m}$ and set it to $\bar{m}=1$. As an aside we remark that in computations of $\mathrm{F}$-terms the parameter $\bar{m}$ plays very much the same role as the the parameter $\beta$ (the inverse temperature) does in the usual heat kernel arguments that compute the anomaly or Witten index (more precisely, $\bar{m} \sim 1 / \beta$ ). One can send $\bar{m}$ either to zero or infinity and thus relate different expansions.

Keeping track of the grassmann integrals also leads to another important restriction valid for F-terms: a diagram with $\ell$ loops will contribute precisely a factor of $\mathcal{W}^{2 \ell}$ (with various possible gauge and spinor index contractions) to the effective superpotential, irrespective of the gauge group and the matter field representations.

\subsection{Loop momentum integrals}

Our task now is to do the actual momentum integrals and compute the Feynman diagrams. Here we take the hint from how the computation of the glueball superpotential is done in the string context [5]. There, in particular, one uses worldsheet moduli, describing the inequivalent conformal structures on the worldsheet. For the string theory argument it is important that one integrates first over the spacetime momenta in the loops and only then over the geometric moduli. The integral over the four-dimensional loop momenta gives a factor

$$
\frac{1}{\operatorname{det}(\operatorname{Im} \Omega)^{2}},
$$

with $\Omega_{a b}$ the period matrix of the Riemann surface that represents the string worldsheet. This measure factor gets cancelled when one does the fermionic momentum integration, as is most easily seen in the Berkovits type formalism applied to this problem in [6]. Thus for these terms in the effective action there is a drastic simplification in the integrand of the string measure on the worldsheet moduli space - the complete dependence on the four-dimensional kinematics disappears. It is this simplification that allows one to reduce the computation to that for a zero-dimensional matrix integral and recover the prescription of [1]. 
In the corresponding field theory setup this suggests that in order to see the simplification one should represent the Feynman amplitude in terms of Schwinger time variables, which are the field theory limits of worldsheet moduli - the Schwinger parameter $s$ associated to a propagator can be thought of as the length of an edge in the Feynman graph.

For any given Feynman graph we have a number of Schwinger time parameters $s_{i}$ where $i$ runs over the edges of the Feynman diagram. We can think of the $s_{i}$ as parametrizing the moduli space of the diagram, i.e. the metric we can put on the graph considered as a one-dimensional (singular) space. They are the QFT analogues of the string worldsheet moduli [7], see also e.g. [8]. In momentum space the propagators are represented as

$$
\int_{0}^{\infty} d s_{i} \exp \left[-s_{i}\left(p_{i}^{2}+\mathcal{W}^{\alpha} \pi_{i \alpha}+m\right)\right]
$$

where we have included the $m$-dependence in the propagator and have put $\bar{m}=1$. Now let $p_{a}$ be the loop momentum for the $a$-th loop, and let

$$
p_{i}=\sum_{a} L_{i a} p_{a}
$$

denote the total momentum flowing through the $i$-th propagator, where we can normalize the matrix entries $L_{i a}$ to $0, \pm 1$. In this notation the Schwinger action for the $i$-th edge is

$$
\exp \left[-s_{i}\left(\sum_{a} L_{i a} p_{a}\right)^{2}\right]
$$

Let us also introduce the matrix $M_{a b}(s)$ given by

$$
M_{a b}(s)=\sum_{i} s_{i} L_{i a} L_{i b}
$$

This matrix naturally appears if we write the total first-quantized action in terms of the loop momenta $p_{a}$ as

$$
\exp \left[-\sum_{a, b} p_{a} M_{a b}(s) p_{b}\right]
$$

We can perform the gaussian integral over bosonic momenta in all loops and obtain

$$
Z_{\text {boson }}=\int \prod_{a=1}^{\ell} \frac{d^{4} p_{a}}{(2 \pi)^{4}} \exp \left[-\sum_{a, b} p_{a} M_{a b}(s) p_{b}\right]=\frac{1}{(4 \pi)^{2 \ell}} \frac{1}{(\operatorname{det} M(s))^{2}}
$$

Thus, the factor $(\operatorname{det} M(s))^{-2}$ gives the measure on the field theoretic Schwinger moduli space. Note that the matrix $M_{a b}$ can be identified as the field theory limit of the period 
matrix $\operatorname{Im} \Omega_{a b}$ in the worldsheet theory, and the above measure is the QFT analogue of (3.5).

For the fermionic momenta we can do something very similar. Let $\pi_{a}^{\alpha},(a=1, \ldots, \ell$, $\alpha=1,2)$ denote the fermionic spinor momentum running around the $a$-th loop. The total $\pi$-momentum flowing through the $i$-th propagator is then given again by

$$
\pi_{i \alpha}=\sum_{a} L_{i a} \pi_{a \alpha}
$$

We now have to perform the grassmann integrals

$$
\int \prod_{a=1}^{\ell} d^{2} \pi_{a} \exp \left[-\sum_{i} s_{i}\left(\sum_{a} \mathcal{W}_{i}^{\alpha} L_{i a} \pi_{a \alpha}\right)\right]
$$

This expression gets multiplied with the relevant group theory factor. Here $\mathcal{W}_{i}^{\alpha}$ means the restriction of $\mathcal{W}^{\alpha}$ to the representation dictated by the $i$-th edge and this should be viewed as an operator on the $i$-th representation vector space. So a more explicit notation would be

$$
\mathcal{W}_{i}^{\alpha}=\sum_{A} \mathcal{W}_{A}^{\alpha} T_{i}^{A}
$$

where $A$ is a Lie algebra index and $T_{i}^{A}$ denotes the corresponding Lie algebra generator in the representation propagating through the $i$-th edge. The evaluations of the term (3.13), i.e. the integration over the fermionic loop momenta $\pi_{a}$, will very much depend on the representation structure of the edges and various group theory factors, which can in principle be analyzed. This will be presented elsewhere [2]. In the next section we see a dramatic simplification for the $U(N)$ case with adjoint representation (or fundamental representations) which verifies the matrix integral proposal of [1].

\section{Proof of the Matrix Integral Reduction for $U(N)$}

For the case of a $U(N)$ gauge theory interacting with a matter field in the adjoint representation (which can be easily generalized to quiver type theories involving product of $U\left(N_{i}\right)$ ) we can use the 't Hooft double line notation to keep track of the gauge index structure. The 't Hooft diagrams at $\ell$ loops have at most $h=\ell+1$ holes (including the outer boundary). 
Suppose now we are interested in computing the superpotential for the traceless glueball field

$$
S=\frac{1}{32 \pi^{2}} \operatorname{Tr}_{S U(N)} \mathcal{W}^{\alpha} \mathcal{W}_{\alpha}
$$

So we explicitly exclude higher order traces of the form $\operatorname{Tr} \mathcal{W}^{n}$ with $n>2$. Since $S$ is given by the quadratic form $\operatorname{Tr} \mathcal{W}^{2}$, this means that in the 't Hooft double line notation we want for each index loop or hole exactly two insertions of $\mathcal{W}$. Thus we need at least $\ell$ index loops, since, as we discussed in the previous section, at $\ell$ loops in field theory we will bring down $2 \ell$ factors of $\mathcal{W}$ in the Schwinger representation of the Feynman diagram. But this can only happen if the diagram is planar, as was already noted in [1]. Planarity is therefore enforced by the fermionic integrals, independent of the precise measure induced by the loop momenta.

Note that there is necessarily one index loop where $\mathcal{W}$ is not inserted. We have $h=\ell+1$ index loops and we have to choose in which index loop we will not insert any $\mathcal{W}$ 's. This can clearly be done in $h$ ways and this gives a combinatorial factor $h$ multiplying the diagram. We also get a factor of $N$ for the single index loop which has no fields attached to it.

Once all this is done we can associate each $\mathcal{W}_{i}$ as being part of a " $\mathcal{W}$-loop." Such an index loop is in general formed by multiplying and finally tracing a series of $\mathcal{W}$ insertions

$$
\operatorname{Tr}\left(\mathcal{W}_{i_{1}} \cdots \mathcal{W}_{i_{n}}\right)
$$

But in the present case, where we only consider the dependence on the bilinear $S=$ $\frac{1}{32 \pi^{2}} \operatorname{Tr} \mathcal{W}^{2}$, such a loop only consists of two $\mathcal{W}$ 's. After we have done the $\mathcal{W}$ insertions, two per index loop with fixed and opposite spinor indices, the complete group index structure is entirely captured by just multiplying each loop by a factor $16 \pi^{2} S$.

Note that quite generally, also for the generating function of traces of higher powers of $\mathcal{W}$, we can have for every edge at most two $\mathcal{W}$ 's inserted. To see this for the $i$-th propagator, we can use the fermionic loop momentum $\pi_{i}$ as one of the fermionic integration variables. Doing the integral $\int d^{2} \pi_{i}$ will then bring down at most two $\mathcal{W}$ insertions.

Furthermore we should recall that the fields are in the adjoint representation of $U(N)$ and therefore the action of $\mathcal{W}$ is through commutators. That is, in the Schwinger action we have the term

$$
\exp \left(-s_{i}\left[W_{i}^{\alpha},-\right] \pi_{i \alpha}\right)
$$

Because of this commutator the insertions at the two double lines come with opposite signs. As we have stated already, for a planar diagram we can identify the index loops 
with the momentum loops (except for one, say the outer loop). Every index loop has a canonically induced orientation coming from the orientation of the plane, and we use the same orientation to assign the direction of the loop momenta. In this way the two lines that make up the double line propagator have opposite orientation. Note that because of the commutator the $\mathcal{W}$ insertions on the left or right index line have signs that are correlated with the index loop orientations.

We can now use this fact, correlating the index loops and momentum loops, to keep track of these group index contractions in a way that is strictly analogous to the fermionic loop momenta $\pi_{a}^{\alpha}$. We will introduce a set of auxiliary grassmannian variables $\mathcal{W}_{a}^{\alpha}$ for each loop and write

$$
\mathcal{W}_{i}^{\alpha}=\sum_{a} L_{i a} \mathcal{W}_{a}^{\alpha}
$$

The cases $L_{i a}= \pm 1$ correspond to the left and right commutator action respectively. Contracting the group indices is now equivalent to performing the fermionic integration over the auxiliary variables $\mathcal{W}_{a}^{\alpha}$. It gives automatically precisely two $\mathcal{W}$ insertions per index loop, and it also assigns the right signs.

This trick allows us to represent the fermionic contribution (3.13) to the Feynman diagram as an integral over the grassmannian loop momenta $\pi_{a}^{\alpha}$ and "index momenta" $\mathcal{W}_{a}^{\alpha}$

$$
\begin{aligned}
Z_{\text {fermion }} & =N h\left(16 \pi^{2} S\right)^{h-1} \int \prod_{a} d^{2} \pi_{a} d^{2} \mathcal{W}_{a} \exp \left[-\sum_{i} s_{i}\left(\sum_{a, b} \mathcal{W}_{a}^{\alpha} L_{i a} L_{i b} \pi_{b \alpha}\right)\right] \\
& =N h\left(16 \pi^{2} S\right)^{h-1} \int \prod_{a} d^{2} \pi_{a} d^{2} \mathcal{W}_{a} \exp \left[-\sum_{a, b} \mathcal{W}_{a}^{\alpha} M_{a b}(s) \pi_{b \alpha}\right] \\
& =N h S^{h-1}(4 \pi)^{2 h-2}(\operatorname{det} M(s))^{2}
\end{aligned}
$$

This should finally be multiplied with the corresponding bosonic measure factor (3.11). Using the planarity relation $\ell=h-1$ that contribution can be written as

$$
Z_{\text {boson }}=\frac{1}{(4 \pi)^{2 h-2}} \frac{1}{(\operatorname{det} M(s))^{2}}
$$

We see that all $s$ dependence cancels between bosons and fermions giving for a diagram with $h$ holes the simple factor

$$
Z_{\text {boson }} \cdot Z_{\text {fermion }}=N h S^{h-1}
$$


This cancellation is the field theory reflection of the fact that we are computing an amplitude that within string theory reduces to a topological string amplitude. Note that the straightforward expansion in $\mathcal{W}$ 's leads to contributions of many different Feynman diagrams - we can distribute the $\mathcal{W}$ 's over the different propagators and within each propagator over the two double lines. The kinematic weight for each of the individual diagrams can be very complicated, which one discovers immediately by computing a specific diagram. Only for the sum of all these diagrams do the fermionic and bosonic measures cancel. Of course, this sum of diagrams is realized by one string worldsheet, and that is why the cancellation argument is so elegant and direct within the string setup. In the field theory limit we localize to various corners of the moduli space and the simplification is less explicit.

With the cancellation of the bosonic and fermionic measures we are thus left with the contribution of the holomorphic mass $m$. For each Schwinger time $s_{i}$ we have the simple integral

$$
\int d s_{i} e^{-s_{i} m}=\frac{1}{m}
$$

This reproduces the propagator of the zero-dimensional action $\frac{m}{2} \operatorname{Tr} \Phi^{2}$. Including also the coupling constants from the vertices we have thus obtained the same amplitude as the planar graphs of a matrix model with $h$ boundaries multiplied by $N h S^{h-1}$ as was proposed in [1]. Hence, we have derived the following formula for the perturbative contribution of the effective glueball superpotential

$$
W_{\text {pert }}(S)=N \frac{\partial \mathcal{F}_{0}}{\partial S}
$$

with

$$
\mathcal{F}_{0}(S)=\sum_{h} \mathcal{F}_{0, h} S^{h}
$$

where $\mathcal{F}_{0, h}$ is the matrix model planar amplitude with $h$ holes. Note that the full answer for the effective superpotential also includes the Veneziano-Yankielowicz term $N S \log \left(S / \Lambda^{3}\right)$ [9], which in the matrix model comes from the volume of the gauge group [10].

We could also be asking about contributions of the form

$$
\frac{1}{2} \int d^{4} x d^{2} \theta \tau(S) \operatorname{Tr} \mathcal{W}^{\alpha} \operatorname{Tr} \mathcal{W}_{\alpha}
$$

This kind of amplitude computes the $U(1)$ coupling constants upon gaugino condensation. In this case, as noted in [1], the 't Hooft index structure is such that on two of the index 


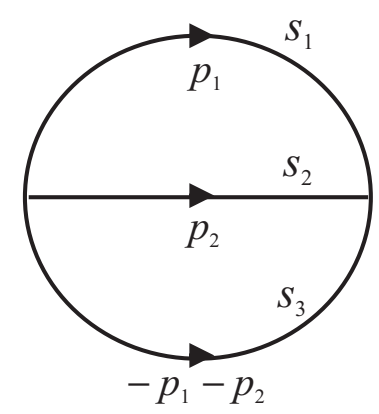

Fig. 1: A two-loop diagram: $s_{i}$ are the Schwinger parameters, the loop momenta flowing through the propagators are indicated.

loops we have to put a single $\mathcal{W}$ while the rest get two each. There are $h(h-1) / 2$ ways to do that. Once this is done the rest of the computation is identical to the one above. Thus in particular we find

$$
\tau(S)=\frac{\partial^{2} \mathcal{F}_{0}}{\partial S^{2}},
$$

as was proposed in [1].

Note that the above computation will also give rise to contributions involving $\mathcal{W}$ with a different index contraction structure. In particular, an $\ell$-loop gauge theory Feynman diagram, when written in double line notation, can correspond to a surface with $g$ handles and $h$ holes as long as

$$
2 g-2+h=\ell-1 .
$$

with the total of $2 \ell$ insertions of $\mathcal{W}$ distributed among the $h$ holes. For such a general amplitude the computation will be similar to the above computation done for planar diagrams. A more natural role for the non-planar matrix model diagrams come from gravitational couplings to RR-field strength which capture the lack of anti-commutativity in the $\theta$-directions for the gauge theory [11].

\subsection{An example}

Just to illustrate how the computation works in a specific case we present a simple example here. Let us consider the model with a single adjoint chiral multiplet and a cubic superpotential

$$
W(\Phi)=\operatorname{Tr}\left(\frac{1}{2} m \Phi^{2}+\frac{1}{6} g \Phi^{3}\right) .
$$

In this model we will now compute what is essentially the first non-trivial diagram the "stop sign" two-loop diagram depicted in fig. 1. In this diagram there are three 


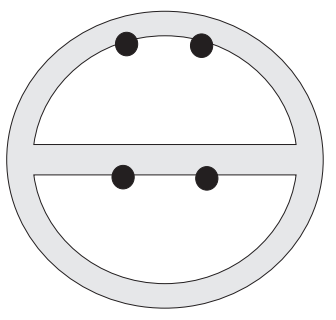

$$
s_{1}{ }^{2} s_{2}^{2}
$$

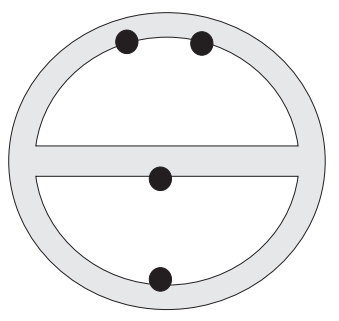

$S_{1}^{2} S_{2} S_{3}$

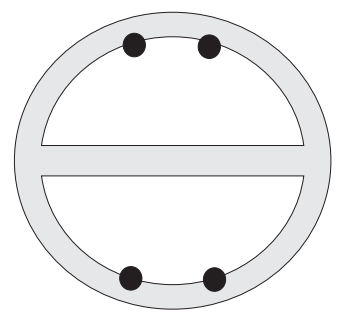

$s_{1}^{2} s_{3}^{2}$

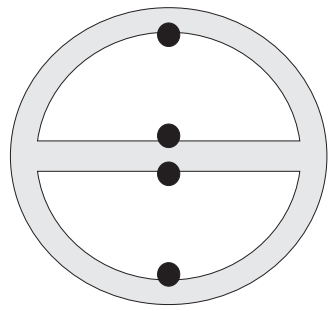

$S_{1} S_{2}^{2} S_{3}$

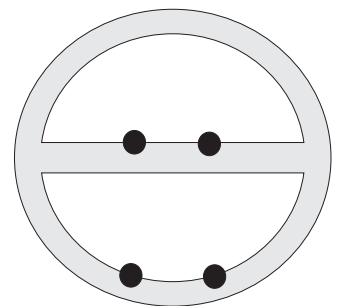

$S_{2}{ }^{2} S_{3}^{2}$

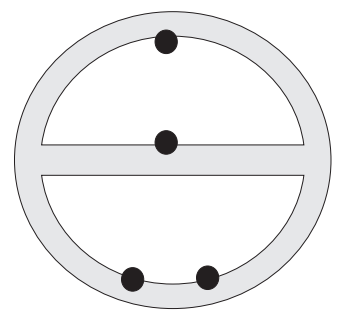

$S_{1} S_{2} S_{3}^{2}$

Fig. 2: The diagrams with $\mathcal{W}$ insertions (indicated by $\bullet$ 's) and the monomials in the Schwinger parameters they compute. We fixed the outer index loop to be free.

propagators with Schwinger lengths $s_{1}, s_{2}, s_{3}$. There are two independent loop momenta $p_{1}, p_{2}$. We will pick a basis such that the bosonic momenta flowing through the three edges is $p_{1}, p_{2},-p_{1}-p_{2}$ respectively, with similar expressions for the fermionic loop momenta $\pi_{i}$. So in this case our matrices $L_{i a}$ and $M_{a b}$ are given by

$$
L_{i a}=\left(\begin{array}{cc}
1 & 0 \\
0 & 1 \\
-1 & -1
\end{array}\right)
$$

and

$$
M_{a b}=\left(\begin{array}{cc}
s_{1}+s_{3} & s_{3} \\
s_{3} & s_{2}+s_{3}
\end{array}\right) .
$$

The integral over the bosonic loop momenta takes the form

$$
\int \frac{d^{4} p_{1}}{(2 \pi)^{4}} \frac{d^{4} p_{2}}{(2 \pi)^{4}} \exp \left[-s_{1} p_{1}^{2}-s_{2} p_{2}^{2}-s_{3}\left(p_{1}+p_{2}\right)^{2}\right],
$$

and this gives the measure factor

$$
\frac{1}{(4 \pi)^{4}} \frac{1}{(\operatorname{det} M(s))^{2}}=\frac{1}{(4 \pi)^{4}} \frac{1}{\left(s_{1} s_{2}+s_{2} s_{3}+s_{3} s_{1}\right)^{2}}
$$


To find the $\mathcal{W}$ dependence we have to evaluate now the fermionic loop momentum integral. Instead of using the representation in our proof, let us compute this "by hand" by summing over all possible $\mathcal{W}$ insertions. An insertion on the $i$-th propagator gives a factor $s_{i}$. There are at most two insertions on one edge.

If we fix one of the index loops to be free of insertions, say the outer loop, then there is precisely one diagram for each monomial in the $s_{i}$ 's, see fig. 2. Note that every factor $s_{i}^{2}$ comes with an extra factor of $\frac{1}{2}$ because we expand the exponential to second order. In this way we find the weight

$$
\frac{3}{4}\left(\operatorname{Tr} \mathcal{W}^{2}\right)^{2}\left(s_{1}^{2} s_{2}^{2}+2 s_{1}^{2} s_{2} s_{3}+\text { permutations }\right)
$$

which can be written as

$$
3 S^{2} \cdot(4 \pi)^{4} \cdot\left(s_{1} s_{2}+s_{2} s_{3}+s_{3} s_{1}\right)^{2}
$$

Note the factor $h=3$ that comes from choosing one of the three index loops on which one does not insert $\mathcal{W}$ 's. This fermionic contribution cancels exactly the momentum integral (4.18), as promised.

\section{Acknowledgements}

We would like to thank J. de Boer, S. Gukov, P. van Nieuwenhuizen and H. Ooguri for valuable discussions. R.D. wishes to thank Harvard University for kind hospitality during this work.

The research of R.D. is partially supported by FOM and the CMPA grant of the University of Amsterdam; M.T.G is supported by NSF grant PHY-0070475; C.S.L. is supported in part by NSERC, Canada and Fonds de Recherche sur la Nature et les Technologies du Quebec; C.V. is partially supported by NSF grants PHY-9802709 and DMS0074329; and D.Z. is partially supported by INFN, MURST, and the European Commission RTN program HPRN-CT-2000-00113. 


\section{References}

[1] R. Dijkgraaf, C. Vafa, "A Perturbative Window into Non-Perturbative Physics," hep-th/0208048.

[2] R. Dijkgraaf, M.T. Grisaru, C.S. Lam, C. Vafa, and D. Zanon, to appear.

[3] S.J. Gates, M.T. Grisaru, M. Roček and W. Siegel, Superspace, Addison Wesley 1983, hep-th/0108200.

[4] M.T. Grisaru and D. Zanon, Nucl. Phys. B252 (1985) 578.

[5] M. Bershadsky, S. Cecotti, H. Ooguri and C. Vafa, "Kodaira-Spencer theory of gravity and exact results for quantum string amplitudes," Commun. Math. Phys. 165, 311 (1994) arXiv:hep-th/9309140.

[6] N. Berkovits and C. Vafa, "N=4 Topological Strings," Nucl. Phys. B 433123 (1995) arXiv:hep-th/9407190.

[7] J. Polchinski, "Evaluation Of The One Loop String Path Integral," Commun. Math. Phys. 104, 37 (1986).

[8] C.S. Lam, "Multiloop String-Like Formulas for QED," Phys. Ref. D 48 (1993) 873 arXiv:hep-ph/9212296.

[9] G. Veneziano and S. Yankielowicz, "An Effective Lagrangian For The Pure $N=1$ Supersymmetric Yang-Mills Theory," Phys. Lett. B 113, 231 (1982).

[10] H. Ooguri and C. Vafa, "Worldsheet derivation of a large $N$ duality," arXiv:hepth/0205297.

[11] H. Ooguri and C. Vafa, work in progress. 